\title{
A New Margin Function for Anti-infective Trials
}

\author{
Félix Almendra-Arao ${ }^{1}$, Hortensia J. Reyes-Cervantes ${ }^{2} \&$ José J. Castro-Alva $^{2}$ \\ ${ }^{1}$ UPIITA del Instituto Politécnico Nacional, México \\ ${ }^{2}$ Benemérita Universidad Autónoma de Puebla, México \\ Correspondence: Félix Almendra-Arao, UPIITA del Instituto Politécnico Nacional, Ciudad de México, México.
}

Tel: (52)-555-729-6000, Ext. 56860. E-mail: falmendra@ipn.mx

\author{
Received: February 3, 2017 Accepted: March 8, 2017 Online Published: April 27, 2017 \\ doi:10.5539/ijsp.v6n3p204 URL: https://doi.org/10.5539/ijsp.v6n3p204
}

\begin{abstract}
In diverse contexts comparison of groups is giving frequently. Particularly, comparison of groups based on non-inferiority statistical tests is becoming more frequent and have had a very special boom in clinical trials, especially in trials related to testing new anti-infective products. Non-inferiority tests are statistical procedures that allow verify whether a sample provides sufficient evidence that the efficacy of a new treatment is not substantially inferior to the known efficacy of a standard treatment. For the selection of the non-inferiority margin for anti-infective trials, the Food and Drug Administration (FDA) and the Committee for Proprietary of Medical Products (CPMP) have provided some general guidance. In this investigation we propose a new parametric family of margin functions for testing non-inferiority in the context of anti-infective trials. One important feature of this parametric family is that fit together recommendations of FDA and CPMP jointly with some other important mathematical properties underlined in this research.
\end{abstract}

Keywords: non-inferiority tests, FDA, Anti-infective trials, non-inferiority guidance.

\section{Introduction}

Because more effective medications are available and there is greater penetration of new drugs, clinical research on new drugs rather than demonstrating the superiority of one drug over another has been directed at trying to demonstrate that a new drug is not inferior to a drug with well-known efficacy and which is known as standard treatment.

Clinical trials designed to demonstrate that a new treatment is not inferior to a standard treatment are known as non-inferiority trials and the corresponding statistical tests are called non-inferiority tests.

Related to non-inferiority trials, there are several features actually under discussion, these features not have a definitive solution; among them highlight one of the steps to design a non-inferiority trial: determination of a pre-specified non-inferiority margin, this is a central, particularly difficult and controversial topic.

Based on conclusions of previous investigations, and investigating in the route of establishing adequate margin functions for non-inferiority we conducted this research in the context of testing non-inferiority for anti-infective trials and propose a family of margin functions to be used specifically in this context.

In a recent article Almendra-Arao, Reyes-Cervantes and Castro-Alva (Almendra-Arao et al., 2016) examined the suggestions of the FDA and CPMP in addition to several known margin functions for non-inferiority in the case of anti-infective trials. These authors established useful properties that should fulfill the non-inferiority margin functions for anti-infective trials in such a way that they comply with the suggestions of the FDA and CPMP as well as possessing suitable mathematical properties and they called these functions FCAT functions.

In this research we continue the work of the authors mentioned above and we propose a family of margin functions, specifically within the scope of anti-infective trials.

\section{The Framework}

Our focus in this work is on non-inferiority clinical trials where the primary outcome is a binary variable. Hence, we are considering a scenario in which two drugs, a new drug and a control or standard drug are to be compared via a dichotomous end point in a randomized study.

Thus, suppose that we have randomly selected $n_{1}$ and $n_{2}$ subjects to receive the control drug and the new one, respectively.

So, let $X_{1}$ and $X_{2}$ be the corresponding two independent random variables with parameters $\left(n_{1}, p_{1}\right)$ and $\left(n_{2}, p_{2}\right)$, 
respectively; $p_{1}$ and $p_{2}$ represent the true response probabilities of the standard and experimental drug, respectively; and also we assume that a higher response is more desirable. In this framework the hypothesis of interest (non-inferiority hypothesis) which we want to prove, is the alternative in the following set of hypotheses

$$
H_{0}: p_{2} \leq g\left(p_{1}\right) \quad \text { vs } \quad H_{1}: p_{2}>g\left(p_{1}\right)
$$

When we select $g$ as the identity function $\left(g\left(p_{1}\right)=p_{1}\right)$ then the alternative hypothesis in (1) above is the hypothesis of superiority of the new treatment against the standard treatment, as in this case we have $H_{1}: p_{2}>p_{1}$.

The function $g$ is called the boundary curve of $H_{0}$ or boundary function.

The function $g$ can be represented in the form $g\left(p_{1}\right)=p_{1}-\delta\left(p_{1}\right)$, the function $\delta$ is called the margin function (Zhang, 2006).

In the following, the domain and image of a given function $f$ will be denoted by $D_{f}$ and $I_{f}$, respectively.

As you would expect, the margin function must be a non-negative function, that is, $\delta\left(p_{1}\right) \geq 0$ for all $p_{1} \in D_{\delta}$. The special case $\delta\left(p_{1}\right) \equiv 0$ leads to the superiority case, and when $\delta\left(p_{1}\right)$ not identically zero on $D_{\delta}$ corresponds properly to the non-inferiority instance.

\section{Proposing a New Margin Function}

As mentioned above, Almendra-Arao et al. (Almendra-Arao et al., 2016) have established properties that they considered as desirable to fulfill any margin function for anti-infective non-inferiority trials in order to satisfy requirements of the FDA and the CPMP guides for non-inferiority trials of anti-infective drugs besides fulfilling some other convenient features. These properties are stated in the following definition.

Definition. A function $g:[0,1] \rightarrow[0,1]$ is an FCAT boundary for non-inferiority if it satisfies the following four properties:

$1 \mathrm{~g}$ is derivable on $[0,1]$.

$20 \leq g\left(p_{1}\right)<p_{1} \forall p_{1} \in(0,1)$.

$3 \delta$ is decreasing on $[.7,1]$.

$4 \mathrm{~g}$ pass close enough to $(0.9,0.8)$.

In this section our goal is to propose boundary functions satisfying the desirable conditions of the FCAT boundary function declared above.

Straight lines in general were considered by Phillips (Phillips, 2003), another natural approach is to use the polynomial curves of the next degree, that is, quadratic curves. The main idea is to construct a curve passing through the points $(r, s)$ and $(1, t)$, with $0 \leq r, s<1$ and $s<t \leq 1$. Since to determine a parabola are necessary three points, and as only two points are fixed, then this will generate a family of parabolas passing through the two specified points.

The coefficients of a parabola $y=a x^{2}+b x+c$ passing through these points accomplish

$$
b=\frac{1}{r-1}\left(a+s-t-a r^{2}\right), \quad c=\frac{1}{r-1}\left(r t+a r^{2}-s-a r\right)
$$

and this has meaning for $r \neq 1$, therefore we propose as boundary function

$$
g\left(p_{1}\right)=a p_{1}^{2}+\frac{1}{r-1}\left(a+s-t-a r^{2}\right) p_{1}+\frac{1}{r-1}\left(r t+a r^{2}-s-a r\right) .
$$

This proposed function $g\left(p_{1}\right)$ corresponds to a general case and surely deserves a more extensive analysis that we will do in this work by space reasons.

Therefore in the next, to illustrate, we will concentrate in the specific and outstanding case when $r=s=0, t=1$, then $b=1-a, c=0$, that is in $g_{a}\left(p_{1}\right)=a p_{1}^{2}+(1-a) p_{1}$ where $a$ is a parameter. Note that for this margin function we have $\delta\left(p_{1}\right)=a p_{1}\left(1-p_{1}\right)$.

\subsection{Checking FCAT Boundary Properties for the New Proposal}

We present some plots of $g_{a}$ corresponding to different values of $a$, in figure 1. It is not true that always this parametric family of functions fulfill conditions of FCAT boundaries for non-inferiority. In the same figure it is observed that for $a=2$, not always $g_{a}\left(p_{1}\right) \in[0,1]$ for $p_{1} \in[0,1]$. The same is true for $a=-2$. Thus, in order that this parametric family of functions represent FCAT boundaries for non-inferiority, it is necessary to analyze restrictions for the parameter a. 


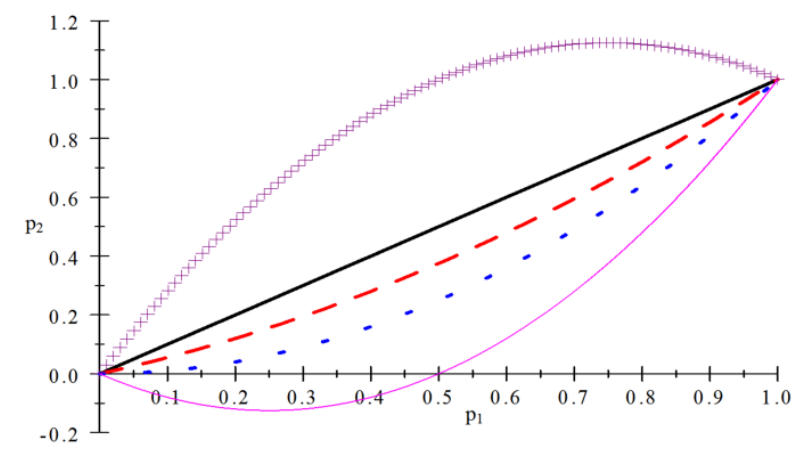

Figure 1. Function $g_{a}\left(p_{1}\right)$. Solid thick: $a=0$, dash: $a=.5$, dots: $a=1$, solid thin: $a=2$, cross: $a=-2$.

We now check the conditions for $a$ such that $g_{a}$ be an FCAT boundary for non-inferiority. $D_{g}=[0,1]$ and image $I_{g} \subseteq[0,1]$, now we want to verify for what values of $a, 0 \leq g\left(p_{1}\right) \leq p_{1}$ for $p_{1} \in D_{g}$.

$0 \leq g_{a}\left(p_{1}\right) \leq p_{1} \forall p_{1} \in[0,1]$, that is to say $0 \leq p_{1}-a p_{1}\left(1-p_{1}\right) \leq p_{1} \forall p_{1} \in(0,1)$ hence $-p_{1} \leq-a p_{1}(1-$ $\left.p_{1}\right) \leq 0 \forall p_{1} \in(0,1)$ thus $0 \leq a p_{1}\left(1-p_{1}\right) \leq p_{1} \forall p_{1} \in(0,1)$ therefore $0 \leq a\left(1-p_{1}\right) \leq 1 \forall p_{1} \in(0,1)$ consequently $0<a<1$.

Additionally when $a=1, g_{a}\left(p_{1}\right)=p_{1}^{2}$ which accomplish also $0 \leq g_{a}\left(p_{1}\right)<p_{1} \forall p_{1} \in(0,1)$.

Thus we have proved that $I_{g_{a}} \subseteq[0,1]$, fulfillment of FCAT1 and FCAT2 it is immediate.

As $\delta^{\prime}\left(p_{1}\right)=a-2 a p_{1}<0$ implies $p_{1}>1 / 2$, this means that property FCAT3 also is accomplished.

Moreover, as the margin function is $\delta\left(p_{1}\right)=a p_{1}\left(1-p_{1}\right)$, then it is immediate that $\delta\left(1-p_{1}\right)=\delta\left(p_{1}\right)$, thus, $\delta$ is symmetric around 0.5 .

Fulfillment of FCAT4 depend of the value of $a$, in the following we analyze performance of this family of margin functions for several values of a.

Röhmel (Röhmel , 2001) noted that the only common point to FDA and CPMP guides is the point $(0.9,0.8)$. Thus in order that $g_{a}\left(p_{1}\right)$ passes through this point we solve $g_{a}\left(p_{1}\right)=a(.9) 2+(1-a) .9=.8$, and we obtain $a=1.1111$. Note that this is not an adequate solution as it does not accomplish the requirement that $a$ belong to the interval $(0,1]$, by this reason it will be discarded.

However, for $a=1$ we have $g_{a}\left(p_{1}\right)=p_{1}^{2}$, thus, $g_{a}(0.9)=(0.9)^{2}=0.81$ which can be considered a good approximation to the common point, as formulated in FCAT 4 property.

Two natural testing procedures obtained using this proposed margin are commented in the next two sections.

\subsection{An Asymptotic Test Obtained via the New Margin Function}

Zhang (Zhang, 2006) derived a class of asymptotic tests based on the delta method for smooth margin functions, the statistic for that asymptotic tests is

$$
T_{S}=\frac{\widehat{p_{2}}-\widehat{p_{1}}+\delta\left(\widehat{p_{1}}\right)}{n_{1}^{-1} \widetilde{p_{1}}\left(1-\widetilde{p_{1}}\right)\left(\delta^{\prime}\left(\widehat{p_{1}}\right)-1\right)^{2}+n_{2}^{-1} \widetilde{p_{2}}\left(1-\widetilde{p_{2}}\right)}
$$

With the family of margin functions proposed in this research, the corresponding test based on Zhang's procedure yields the following family of statistics

$$
T_{a}=\frac{\widehat{p_{2}}-\widehat{p_{1}}+a \widehat{p_{1}}\left(1-\widehat{p_{1}}\right)}{n_{1}^{-1} \widetilde{p_{1}}\left(1-\widetilde{p_{1}}\right)\left(a-1-2 a \widetilde{p_{1}}\right)^{2}+n_{2}^{-1} \widetilde{p_{2}}\left(1-\widetilde{p_{2}}\right)}
$$

one statistic for each value of $a \in(0,1]$, here $\widehat{p_{1}}, \widehat{p_{2}}$ are the maximum likelihood estimators (MLE) and $\widetilde{p_{1}}, \widetilde{p_{2}}$ are the MLE restricted under the null hypothesis.

\subsection{The Barnard Exact Test using the New Margin Function}

The procedure corresponding to the exact Barnard's test (Barnard, 1945; Barnard, 1947) can be naturally extended to non-inferiority. 
Roughly speaking, Barnard's procedure consists in iteratively computing the test size in order to construct the critical region, taking as initial region the set $\left\{\left(0, n_{2}\right)\right\}$ and incorporating points according to the criterion of order proposed by Barnard (Barnard, 1945).

In a recent article of Almendra-Arao, Sotres-Ramos and Zúñiga-Estrada (Almendra-Arao, Sotres-Ramos, \& Zúñiga-Estrada, 2017), was studied the Barnard's exact test and some theoretical properties were obtained. In that work, authors take the idea behind the Barnard's procedure and applied it to the context of non-inferiority for any non-decreasing margin function $g:[0,1] \rightarrow[0,1]$.

Note that if $g$ is a FCAT function the Barnard's procedure can be extended as described by Barnard (Barnard, 1947). Thus, in particular, Barnard's procedure can be used with the margin function proposed in this work

$$
g\left(p_{1}\right)=a p_{1}^{2}+\frac{1}{r-1}\left(a+s-t-a r^{2}\right) p_{1}+\frac{1}{r-1}\left(r t+a r^{2}-s-a r\right)
$$

Particularly with the function $g_{a}\left(p_{1}\right)=a p_{1}^{2}+(1-a) p_{1}$.

\section{Comparison of the New Proposal with Some useful Non-inferiority Margin Functions}

As seen in Almendra-Arao et al. (Almendra-Arao et al., 2016), difference between proportions, the FDA step function as well as the proportions ratio are not very useful dissimilarity measures from the point of view under consideration in this work. For that reason, we will not compare the new proposal with those. However in that work was noted that among the analyzed boundary functions, the more convenient were the odds ratio, a particular case of Phillips's linear margin and Röhmel's proposals.

In the following subsections we compare the proposed family of boundary functions in this work $g_{a}$ with odds ratio, a particular case of Phillip's linear margin and Röhmel's proposals, we made this comparison by doing that the difference between the areas of the corresponding margin functions be equal to zero.

These mentioned boundary functions are the following

Odds ratio: $g\left(p_{1}\right)=\frac{p_{1}}{O_{0}+\left(1-O_{0}\right) p_{1}}$ where $O_{0}$ is a constant with $O_{0} \geq 1$.

Phillip's linear: $g\left(p_{1}\right)=(1-b) p_{1}-\gamma$ (Phillips, 2003).

Röhmel's proposals

$g_{R_{1}}\left(p_{1}\right)=p_{1}-.333 \sqrt{p_{1}\left(1-p_{1}\right)}$ (Röhmel, 2001).

$g_{R_{2}}\left(p_{1}\right)=p_{1}-.223 \sqrt[3]{p_{1}\left(1-p_{1}\right)}$ (Röhmel, 2001).

$g_{R_{3}}\left(p_{1}\right)=\Phi\left(\Phi^{-1}\left(\mathrm{p}_{1}\right)-\mathrm{d}\right)$ (Röhmel, 1998), where $d$ is a constant and $\Phi$ is the standard normal distribution function.

Then to determine convenient values for $a$, it seems a reasonable approach to take those boundaries in account. In the next sections we force the areas between those functions and $g_{a}$ equal to zero.

\subsection{Difference between Areas to Odds Ratio}

As was noted by Almendra-Arao et al. (Almendra-Arao et al., 2016) odds ratio dissimilarity measure behaves well from the point of view considered in that work, additionally for $O_{0}=2.25$ the boundary function passes through the common point suggested by FDA and CPMP, therefore we use this value of $O_{0}$ to compare. Thus we have that

$$
\begin{aligned}
&\left|\int_{0}^{1}\left(g_{a}\left(p_{1}\right)-g_{O_{0}}\left(p_{1}\right)\right) d p_{1}\right|=\left|\int_{0}^{1}\left(a p_{1}^{2}-a p_{1}+\frac{p_{1}\left(1-p_{1}\right)(2.25-1)}{p_{1}+2.25\left(1-p_{1}\right)}\right) d p_{1}\right| \\
&=|0.13226-0.16667 a|=0
\end{aligned}
$$

the solution is $a=0.79354$ and for this value of $a, g_{a}(0.9)=0.82858$, in other words, $g_{a}$ cross the point $(0.9,0.82858)$, which is not very far from the suggested point by FDA and CPMP.

In figure 2 we illustrate these two boundary functions. 


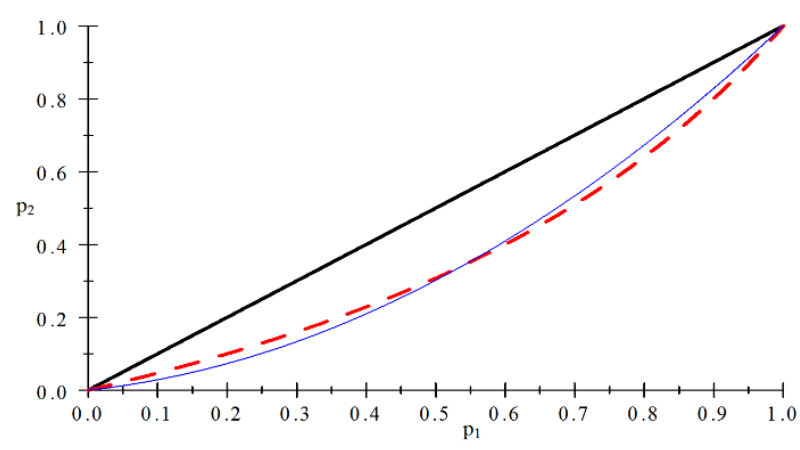

Figure 2. Solid thick: identity function, dash: odds ratio boundary for $O_{0}=2.25$, solid thin: $g_{a}$ for $a=0.79354$.

\subsection{Difference Between Areas to Phillips's Linear Margin}

As discussed in Almendra-Arao et al. (Almendra-Arao et al., 2016) the linear margin passing through $(0, t)$ and $(0.9,0.8)$ is

$$
g\left(p_{1}\right)=\left(1-\frac{10 t-1}{10 t-9}\right) p_{1}+\frac{8 t}{10 t-9}
$$

note that $D_{g}=[t, 1]$. Now computing the difference between the areas of this boundary and $g_{a}$ we have

$$
\begin{aligned}
& \left|\int_{t}^{1}\left(a p_{1}^{2}+(1-a) p_{1}-\left(\left(1-\frac{10 t-1}{10 t-9}\right) p_{1}+\frac{8 t}{10 t-9}\right)\right) d p_{1}\right| \\
= & \frac{1}{6|10 t-9|} \mid(t-1)\left(21 t-9 a+28 a t^{2}-20 a t^{3}+a t-30 t^{2}+3 \mid=0\right.
\end{aligned}
$$

then $a=\frac{-30 t^{2}+21 t+3}{20 t^{3}-28 t^{2}-t+9}$

Analyzing performance of the function $\frac{-30 t^{2}+21 t+3}{20 t^{3}-28 t^{2}-t+9}$ which is increasing on $[0,0.5]$, considering that convenient values of $t$ are $\leq 0.3$ and for $a$, adequate values are close to 1 and taking in account the values in Table 1 .

Table 1. Values of $g_{a}(0.9)$ for different values of $a$ and $t$.

\begin{tabular}{lllllll}
\hline$t$ & 0.10 & 0.15 & 0.20 & 0.25 & 0.30 & 0.31 \\
\hline $\mathrm{a}$ & 0.55556 & 0.66063 & 0.76531 & 0.87179 & 0.98214 & 1.0048 \\
\hline$g_{a}(0.9)$ & 0.85 & 0.84954 & 0.83112 & 0.82154 & 0.81161 & 0.80957 \\
\hline
\end{tabular}

The value $t=0.3$ and therefore $a=0.98214$ may be considered as reasonably acceptable, besides the boundary function is $g\left(p_{1}\right)=1.3333 p_{1}-0.4$.

In Figure 3 we plot this specific Phillip's linear margin obtained above and the corresponding $g_{a}$. 


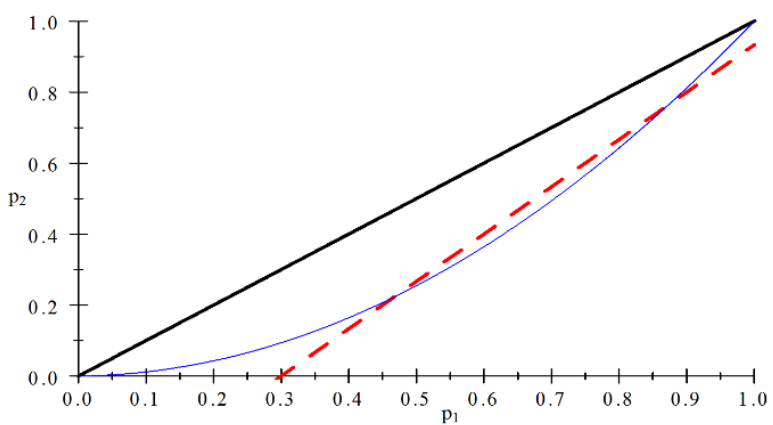

Figure 3. Solid thick: identity function, dash: Phillips's boundary $1.333 p_{1}-0.4$, solid thin: $g_{a}$ for $a=0.98214$.

Note that the corresponding $g_{a}$ boundary pass through the point $(0.9,0.81161)$ which is a good approximation to the target point $(0.9,0.8)$.

Hence we consider this value of $a$ as suitable having in account fulfillment of FCAT4.

\subsection{Difference between Areas to Röhmel's Proposals}

Almendra-Arao et al. (Almendra-Arao et al., 2016) have also noted that the other dissimilarity measures that behave well are the Röhmel's proposals. In this section we compare the family of boundary functions proposed in this work with Röhmel's proposals, doing these comparisons in the same way as in previous section, that is, by setting the difference between the areas of the corresponding margin functions equal to zero.

For the $g_{R_{1}}$ we have

$$
\begin{aligned}
& \left|\int_{0.1}^{1}\left(g_{a}\left(p_{1}\right)-g_{R_{1}}\left(p_{1}\right)\right) d p_{1}\right|=\left|\int_{0.1}^{1}\left(a p_{1}^{2}-a p_{1}+\frac{1}{3} \sqrt{p_{1}\left(1-p_{1}\right)}\right) d p_{1}\right| \\
= & \mid\left(\frac{\pi}{48}-\frac{1}{6} a\right)-\left(-0.0046667 a-0.058637|=| \frac{\pi}{48}-0.162 a+0.058637 \mid=0\right.
\end{aligned}
$$

obtaining $a=0.76597$. Note that $g_{0.76597}(.9)=0.83106$, thus the corresponding point is not far from the target point $(0.9,0.8)$.

In Figure 4 we plotted these two margin functions

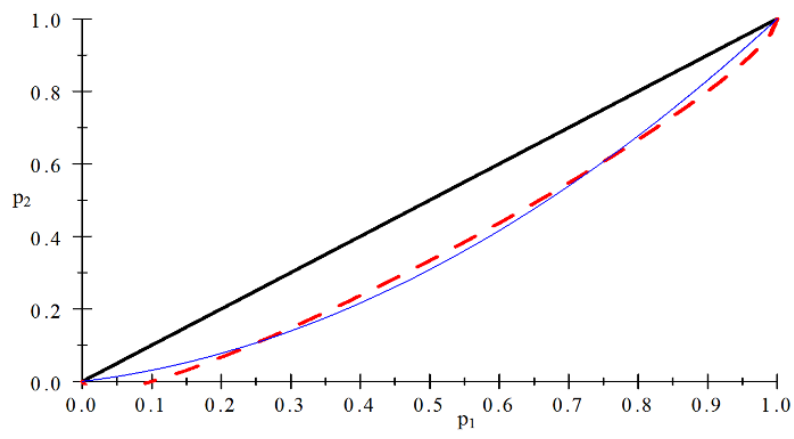

Figure 4. Solid thick: identity function, dash: $g_{R_{1}}$, solid thin: $g_{a}$ for $a=0.76597$.

Whereas that for $g_{R_{2}}$ we have

$$
\left|\int_{0.1}^{1}\left(g_{a}\left(p_{1}\right)-g_{R_{2}}\left(p_{1}\right)\right) d p_{1}\right|=\left|\int_{0.1}^{1}\left(a p_{1}^{2}-a p_{1}+\frac{223}{1000} \sqrt[3]{p_{1}\left(1-p_{1}\right)}\right) d p_{1}\right|
$$




$$
=|-0.162 a+0.11058|=0 \text {, then } a=0.68259 .
$$

In this case $g_{0.68259}(.9)=0.83857$, again the obtained point is not far from the target point $(0.9,0.8)$. In Figure 5 we plotted these two margin functions.

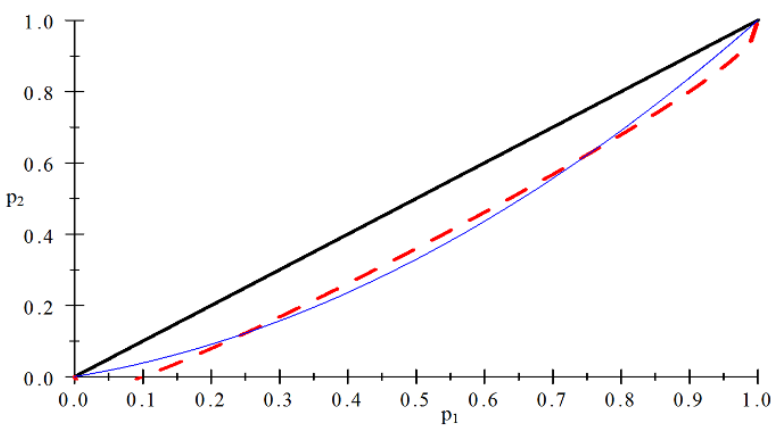

Figure 5. Solid thick: identity function, dash: $g_{R_{2}}$, solid thin: $g_{a}$ for $a=0.68259$.

Finally, for $g_{R_{3}}$ we have

$$
\begin{gathered}
\left|\int_{0}^{1}\left(g_{a}\left(p_{1}\right)-g_{R_{3}}\left(p_{1}\right)\right) d p_{1}\right|=\left|\int_{0}^{1}\left(a p_{1}^{2}+(1-a) p_{1}-\Phi\left(\Phi^{-1}\left(p_{1}\right)-0.43994\right)\right) d p_{1}\right| \\
=\left|\frac{1}{2}-\frac{1}{6} a-0.37787\right|=0, \text { then } a=0.73278 .
\end{gathered}
$$

For this case $g_{0.73278}(.9)=0.83405$, and we can consider that the obtained point is not far from the target point $(0.9,0.8)$. In Figure 6 we plotted the $g_{R_{3}}$ and the new proposal of this investigation.

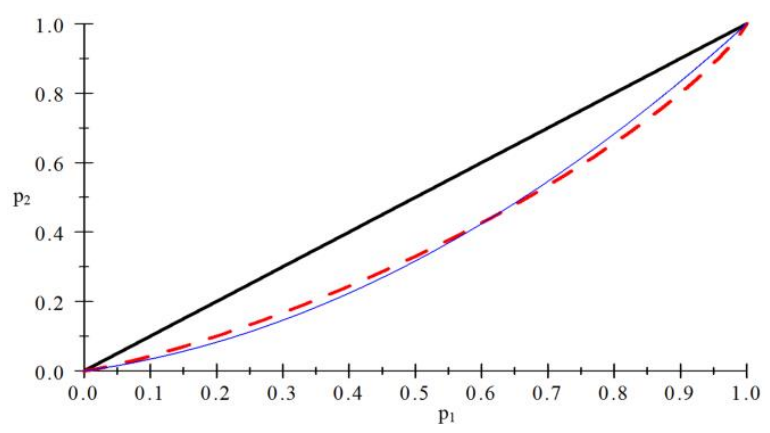

Figure 6. Solid thick: identity function, dash: $g_{R_{3}}$, solid thin: $g_{a}$ for $a=0.73278$.

For the three cases analyzed in this subsection, the obtained points are not as close to the point $(.9, .8)$ as for $a=1$ but they can be considered also appropriated.

\section{Conclusions}

From the previous sections we can extract the following useful information.

In the general situation we posed the boundary function

$$
g\left(p_{1}\right)=a p_{1}^{2}+\frac{1}{r-1}\left(a+s-t-a r^{2}\right) p_{1}+\frac{1}{r-1}\left(r t+a r^{2}-s-a r\right)
$$

which passes through the points $(r, s)$ and $(1, t)$, with $0 \leq r, s<1$ and $s<t \leq 1$. We have analyzed performance of this function for $r=s=0, t=1$, an analysis for cases when $r \neq 0, t \neq 1$ could conduit to fruitful conclusions, however by space reasons we put off this analysis for other work.

For $r=s=0, t=1$, the boundary function that we propose in this work is $g_{a}\left(p_{1}\right)=a p_{1}^{2}+(1-a) p_{1}$, it fulfill 
FCAT1-FCAT3 and symmetry of margin function around 0.5. Fulfillment of FCAT4 depend of the value of $a$. In Table 2, for some values of $a$, are showed the corresponding point through which passes the boundary function

Table 2. Values of $g_{a}\left(p_{1}\right)$ for different values of $a$ and $p_{1}$.

\begin{tabular}{lllllllll}
\hline$a$ & 1 & 1 & .99 & .99 & .90 & .80 & .73278 & .70 \\
\hline$p_{1}$ & .90 & .89 & .90 & .89 & .90 & .90 & .89 & .90 \\
$g_{a}\left(p_{1}\right)$ & .81 & .7921 & .8109 & .79308 & .819 & .828 & .81286 & .837 \\
\hline
\end{tabular}

In this paper we have proposed a parametric family of margin functions $g_{a}$ for $0<a \leq 1$ and we have shown that this family has very convenient properties.

For contrasting two antibacterial products in the presence of a gold standard, we consider useful margin functions: proportions ratio, particularly for $O_{0}=2.25$; Phillips's linear margin, particularly $g\left(p_{1}\right)=1.3333 p_{1}-0.4$ or some other straight line "close" to it; Röhmel's proposals as well as the margin functions proposed in the present article, particularly for values of $a \in[0.7,1]$.

Corresponding to this proposed parametric family of margin functions are associated asymptotic tests based on the delta method by Zhang (Zhang, 2006), analysis of the performance of these tests is awaiting, it is recommendable to include in this analysis the fact that the critical regions must be a Barnard convex sets, see for example Almendra-Arao et al. (Almendra-Arao, et al.,2017) and Almendra-Arao and Sotres-Ramos (Almendra-Arao and Sotres-Ramos, 2014). Also the extended Barnard's procedure can be used with these proposals. In a forthcoming paper, we will study the performance of the statistics yielded from this family of boundary functions.

To have a family of functions represent an advantage because it is possible to select among many of them, in this case among infinite functions. However more research should be done for each specific context in order to determine the more convenient margin function for such context.

Among other advantages of this family of margin functions it is noticeable that they are easily derivable. This is a very useful property if for example it is necessary to derivate twice in order to apply the results by Almendra-Arao (Almendra-Arao, 2011).

According to the previous analysis, the choice of the parameter $a$ depend of several features of the experiment and to take a final decision will be necessary to consider all involved aspects, statistical and clinical, of the specific trial.

\section{Acknowledgement}

First author want to thanks partial support to this research by SNI-CONACYT, COFAA-IPN, EDI-IPN and Project SIP-IPN 20160687.

\section{References}

Almendra-Arao, F. (2011). Barnard convex sets. Communications in Statistics-Theory and Methods, 40(14), $2574-2582$. https://doi.org/10.1080/03610926.2010.484155

Almendra-Arao, F., \& Sotres-Ramos, D. (2014). On the importance in clinical trials that critical regions for comparing 2 independent proportions must be Barnard convex sets. Therapeutic Innovation \& Regulatory Science, 48(2), 208-212. https://doi.org/10.1177/2168479013500968

Almendra-Arao, F., Reyes-Cervantes, H., \& Castro-Alva, J. (2016). Construccting convenient boundary functions for anti-infective trials. JP Journal of Biostatistics, 13(2), 135-154. https://doi.org/10.17654/BO013020135

Almendra-Arao, F., Sotres-Ramos, D., \& Zúñiga-Estrada, M. (2017). Extending the exact Barnard's test to non-inferiority. Communications in Statistics-Theory and Methods., 46(13) 6293-6302 https://doi.org/10.1080/03610926.2013.875577.

Barnard, G. (1945). A new test for 2x2 tables. Nature, 156, 783-784. https://doi.org/10.1038/156783b0

Barnard, G. (1947). Significance tests for 2x2 tables. Biometrika, 34, 123-138.

Phillips, K. (2003). A new test for non-inferiority for anti-infective trials. Statistics in Medicine, 22, 201-212. https://doi.org/10.1002/sim.1122

Röhmel, J. (1998). Therapeutic equivalence investigations: statistical considerations. Statistics in Medicine, 17:1703-1714. https://doi.org/10.1002/(SICI)1097-0258(19980815/30)17:15/16<1703::AID-SIM972>3.0.CO;2-G 
Röhmel, J. (2001). Statistical considerations of FDA and CPMP rules for the investigation of new antibacterial products. Statistics in Medicine, 20:2561-2571. https://doi.org/10.1002/sim.729

Zhang, Z. (2006). Non-inferiority testing with a variable margin. Biometrical Journal, 48 (6), 948-965.

\section{Copyrights}

Copyright for this article is retained by the author(s), with first publication rights granted to the journal.

This is an open-access article distributed under the terms and conditions of the Creative Commons Attribution license (http://creativecommons.org/licenses/by/4.0/). 